\title{
Enhancement of tendon-bone interface healing and graft maturation with cylindrical Titanium-Web (TW) in a miniature swine anterior cruciate ligament reconstruction model: Histological and collagen- based analysis
}

Keisho Ryu ( $\nabla$ rkeisho@gmail.com )

Tokyo Jikeikai Ika Daigaku https://orcid.org/0000-0002-8851-449X

Mitsuru Saito

Tokyo Jikeikai Ika Daigaku

Daisaburo Kurosaka

Tokyo Jikeikai Ika Daigaku

Seiichiro Kitasato

Tokyo Jikeikai Ika Daigaku

Toshiyuki Omori

Tokyo Jikeikai Ika Daigaku

Hiroteru Hayashi

Tokyo Jikeikai lka Daigaku

Tomohiro Kayama

Tokyo Jikeikai Ika Daigaku

Keishi Marumo

Tokyo Jikeikai lka Daigaku

\section{Research article}

Keywords: ACL reconstruction, enthesis, tendon-bone interface, titanium-web, miniature swine, collagen maturation

Posted Date: December 18th, 2019

DOI: https://doi.org/10.21203/rs.2.19196/v1

License: (c) (1) This work is licensed under a Creative Commons Attribution 4.0 International License.

Read Full License 
Version of Record: A version of this preprint was published at BMC Musculoskeletal Disorders on March 31st, 2020. See the published version at https://doi.org/10.1186/s12891-020-03199-0. 


\section{Abstract}

Background: Tendon-bone interface healing and ligamentization of the graft in anterior cruciate ligament (ACL) reconstruction with autografts are important factors affecting treatment outcome. This study aimed to investigate the effectiveness of a cylindrical titanium-web (TW) in tendon-bone interface healing and graft maturation in ACL reconstruction.

Methods: Fourteen mature female CLAWN miniature swine underwent bilateral ACL reconstructions with patellar tendon (PT) autografts. In one limb, the TW/tendon complex was placed into the proximal side of the tibial tunnel. Only the graft was transplanted into the tunnel in the control limb. The proximal side of the graft was sutured into the stump of the native ACL and the distal end was stapled to the tibia. The animals were euthanized at 4 and 15 weeks postoperatively, for histological and biochemical analyses.

Results: Microscopic images in TW limbs showed that ingrowth of tendon-like tissue and mineralized bone tissue into the TW connected the bone and the tendon directly. In contrast, fibrous tissue intervened between the bone and tendon in the control limbs. The total amount of collagen cross-links (which defines the strength of collagen fibers) and the maturation of collagen cross-links in TW tendons were significantly higher $(p<0.05)$ than those of control limbs. There was no significant difference in the ratio of dihydroxy-lysinonorleucine to hydroxy-lysinonorleucine (an indicator of tissue specific collagen maturation) between TW tendons and that of the native PT.

Conclusions: TW promoted the maturation and formation of collagen cross-links in the grafted tendon while maintaining the cross-link pattern of native tendon collagen, and enabled direct binding of tendon to bone.

\section{Background}

Anterior cruciate ligament (ACL) injury is one of the most common knee injuries in teenagers and young adults. Approximately 250,000 individuals, in the USA alone, suffer from ACL injury per year [1]. Usually ACL reconstruction with autografts or allografts is performed to restore function after the injury, and around 175,000 ACL reconstruction surgeries were performed in the USA annually[2]. However, delayed sports recovery and risk of re-rupture remain problematic, and many researches attempt to recreate the native ACL. Tendon-bone interface healing and ligamentization of the graft in Anterior cruciate ligament $(A C L)$ reconstruction with autografts are important factors affecting treatment outcome after surgery [3, 4]. After ACL reconstruction with a free-tendon graft, collagen fibers are first formed at the tendon-bone interface, and then collagen fibers perpendicular to the interface called the Sharpey-like fibers form to connect the tendon and bone tunnel $[5,6]$. This so-called indirect insertion is different from the direct insertion found in the normal ACL tendon-bone interface or tendon-bone late healing phase at the exit of the bone tunnel $[5,7]$. Direct insertions are characterized by connection of the graft and bone tunnel through the fibrocartilage tissue, forming the "tidal line" structure that stains positively for basophilia [5, 7]. Most researchers believe that indirect insertions are less effective than direct insertions and this slow 
and incomplete healing of the tendon-bone interface may result in inferior functional recovery and even worse osteoarthritic changes [8].

The transplanted tendon into the knee joint during ACL reconstruction undergoes a biological transformation process with changes in vasculature and collagen profiles. This remodeling process into a viable ACL-like tissue is termed "ligamentization" [4, 9]. The time required for ligamentization after ACL reconstruction has been reported to range from 9 to 18 months under light microscopy [4], 13 to 31 months under electron microscopy [4], and within one year in a collagen cross-links analysis [10]. The current challenge is to find better strategies to improve tendon-bone interface healing and maturation of the grafted tendon for faster recovery.

Mesenchymal stem cell transplantation [11-14] and administration of biological growth factors, such as recombinant human bone morphogenetic protein-2 (rhBMP-2) $[6,14,15]$, transforming growth factor (TGF) -beta1 [16], fibroblast growth factor (FGF) [5] and granulocyte colony stimulating factor (G-CSF) [17], have been performed in recent studies. Though most studies using growth factors failed to form fibrocartilage tissue at the tendon-bone interface, Hashimoto et al. reported that they succeeded in generating a tendon-bone junction similar to normal enthesis using rhBMP-2 in a rabbit model [15]. However, this method included injecting rhBMP-2 into the semitendinosus tendon to induce ectopic ossicle formation, which was then surgically transferred into the femur and tibia. [6]. This method has problems in clinical application, such as the biological effects caused by growth factors and newly recruited mesenchymal stem cells, as well as high cost, and cumbersome surgical procedure.

In this research, we focused on titanium-web (abbreviated as TW) to promote the anchoring of tendon to bone and induce graft maturation. TW is an unwoven mesh composed of thin titanium fibers, whose individual cross-section is square-shaped with widths of 50-80 $\mu \mathrm{m}$, and the inter-fibril space of 200$500 \mu \mathrm{m}$ [18]. TW has been reported to promote bone formation and has been used as a three-dimensional scaffold for repairing bone defects in previous studies, due of its perfect biocompatibility, good intensity and excellent strength [18-20]. Previously, we implanted a TW to subcutaneous tissue and confirmed the promotion of collagen synthesis growing into the TW (Fig. 1). From the above preliminary experiment, we hypothesized that transplanting the TW/tendon complex into the bone tunnel in ACL reconstruction, will promote bone and tendon-like tissue induction into the TW, thereby anchoring the tendon to bone directly. We also hypothesized that TW will have a positive effect on maturation of transplanted tendon.

The purpose of this study was to investigate the effectiveness of a cylindrical TW in tendon-bone interface healing and graft maturation in ACL reconstruction by histological and biochemical analyses. Among the available experimental animals, miniature swine were selected in this study because they are of sufficient size to perform ACL reconstructions with TW and have a weight that is closer to humans.

\section{Methods}

\section{Biomaterials}


The cylindrical TW (titanium-web) with three-dimensional porous structure (outer diameter $6 \mathrm{~mm}$, inner diameter $3 \mathrm{~mm}$, length $10 \mathrm{~mm}$ ) consisted of $50 \mu \mathrm{m}$ diameter unalloyed titanium fibers (equipped to ASTM B265 Grade1), with $87 \%$ porosity and average $200 \mu \mathrm{m}$ pore space was provided by Hi-Lex Corporation (Hyogo, Japan) for this study (Fig. 2).

\section{Animals}

Fourteen healthy mature female CLAWN miniature swine, 30-33 months old and weighing $36 \pm 10 \mathrm{~kg}$ were obtained from the Kagoshima Miniature Swine Research Center (Kagoshima, Japan). These animals were housed in the animal center of Hamri Co., Ltd (Ibaraki, Japan) under an air-conditioned environment (room temperature: $20 \pm 5^{\circ} \mathrm{C}$, humidity: $55 \pm 15 \%$, lighting time: 12 hours a day). They were fed in pens with free access to adequate food (400-600 g pellet feed for mini-pigs per day) and water.

\section{Surgical procedure}

After intra-muscular administration of ketamine hydrochloride $(10 \mathrm{mg} / \mathrm{kg})$ and xylazine $(4 \mathrm{mg} / \mathrm{kg})$, each animal was fixed in a supine position on a surgical bed. Bilateral ACL reconstructions with patellar tendon (PT) autograft were carried out under the same anesthesia. An anterior longitudinal skin incision $4 \mathrm{~cm}$ in length and medial parapatellar arthrotomy were performed to expose the knee joint. The entire ACL was identified by removing the infra-patellar fat pad. After freeze-processing over the full-length of the ACL using liquid nitrogen, the ligament was disconnected from its tibial attachment and the ligamentbone junction of the proximal tibia was removed completely. A graft width of $3 \mathrm{~mm}$ for ACL reconstruction was harvested from the medial part of the patellar tendon, and disconnected at the edge of the patella. In the TW (titanium-web) limb (right knee), a $6 \mathrm{~mm}$ diameter tibial tunnel was created slightly inwards from the original ACL attachment. After attaching the graft inside TW (Fig. 3a), the TW/tendon complex was placed in the tibial tunnel. In the control limb, only the $3 \mathrm{~mm}$ graft was transplanted into the tibial tunnel. The proximal side of the graft was sutured using a $4-0$ nylon yarn into the stump of the native ACL (Fig. 3b), and was fixed distally with a staple (width $5.5 \mathrm{~mm}$, Daidohant, Osaka, Japan) to the tibia. The joint capsule and skin were closed with 2 - 0 Vicryl suture (Ethicon, Somerville, N.J., USA). No external fixation was performed and plain radiographs of the bilateral knee were taken (Fig. 4). Postoperatively, these animals were allowed to move freely in their pens. They received painkillers ( $20 \mu \mathrm{g} / \mathrm{kg}$ of Buprenorphine) and antibiotics ( $5 \mathrm{mg} / \mathrm{kg}$ of Enrofloxacin) for 7 days. The animals were euthanized at $4(n=4)$ and $15(n=10)$ weeks postoperatively by overdose of pentobarbital sodium ( $60 \mathrm{mg} / \mathrm{kg}$ or more). All Surgical procedure were performed in the operating room of the animal center of Hamri Co., Ltd (Ibaraki, Japan). Histological and biochemical analyses were performed by blinded observers.

\section{Histological analysis}

At 4 and 15 weeks postoperatively (four animals from each group), the proximal end of the tibia with the transplanted graft was resected en bloc and fixed in $4 \%$ paraformaldehyde $(\mathrm{pH} \mathrm{7.3)}$, dehydrated in $70-$ $99.5 \%$ ethanol and acetone for 2 days, exposed to a 1:1 ratio of acetone and methyl methacrylate (MMA) 
monomer for 3 days, further treated with MMA monomer embedded in MMA resin for 10 days, and hardened for 12 days. After polymerization, specimens were cut to a width of 200-300 $\mu \mathrm{m}$ with a microtome (BS-300CL, EXAKT, Hamburg, Germany), and ground to a thickness of 40-50 $\mu \mathrm{m}$ (MG-400CS, EXAKT, Hamburg, Germany).

Microscopic sections were de-plasticized with xylene for $2 \mathrm{~h}$ at $50^{\circ} \mathrm{C}$. De-plasticized sections were stained by Cole's hematoxylin with a $1 \%$ hydrochloric acid-ethanol solution for $60 \mathrm{~min}$, stained by eosin for $3 \mathrm{~min}$, and washed with 99.5\% ethanol (hematoxylin-eosin staining). De-plasticized sections also were stained by iron hematoxylin with a $1 \%$ hydrochloric acid-ethanol solution for $60 \mathrm{~min}$, stained by Ponceau fuchsin with $1 \%$ acetic acid for 10 min, and stained with a phosphotungstic acidphosphomolybdic acid solution. Then they were stained with naphthol green for 30 min and washed with 70-99.5\% ethanol (Villanueva's Goldner staining). In addition, Toluidine blue staining was performed on the de-plasticized Sect. 15 week after surgery. These stained sections were enclosed and observed with a light microscope (BX53, Olympus, Tokyo, Japan).

\section{Biochemical analysis}

Specimens were collected from the transplanted grafts and the lateral part of the patellar tendon in both limbs of miniature swine 15 weeks postoperatively $(n=6)$. Quantitative analysis of divalent immature and trivalent mature collagen cross-links was performed by high-performance liquid chromatography (HPLC) using a fluorescence detection method established previously [21]. Each tissue was minced and suspended in $500 \mathrm{vol}(\mathrm{v} / \mathrm{wt})$ of $0.05 \mathrm{M}$ potassium phosphate buffer, $\mathrm{pH} 7.6$ (ionic strength $=0.15 \mathrm{M}$ ) at $4{ }^{\circ} \mathrm{C}$ and continuously stirred for $72 \mathrm{~h}$ under a vacuum. A $1 / 30$ volume of sodium borohydride ( $\left.\mathrm{NaBH} 4\right)$ was added to the solution. The reaction was allowed to proceed for $60 \mathrm{~min}$ at $37^{\circ} \mathrm{C}$ and was terminated by the addition of $6 \mathrm{~N}$ hydrochloric acid to decrease the $\mathrm{pH}$ value to 4.0. The solution residue was collected by centrifugation ( $3,000 \mathrm{~g}$ for $15 \mathrm{~min}$ ), washed twice with deionized water, and lyophilized. For HPLC analysis, lyophilized samples were dissolved in $0.2 \mathrm{~N}$ sodium citrate buffer $(\mathrm{pH} 2.2)$ and filtered through a $0.45-\mu \mathrm{m}$ filter (Gelman Science Japan Ltd, Tokyo, Japan).

The immature cross-links, such as dihydroxy-lysinonorleucine (DHLNL) and hydroxy-lysinonorleucine (HLNL) were detected by online post column derivatization using 0-phthalaldehyde and were simultaneously monitored by fluorescence with excitation at $350 \mathrm{~nm}$ and emission at $450 \mathrm{~nm}$. Mature pyridinium cross-links, such as pyridinoline (PYD) and deoxypyridinoline (DPD) were detected by natural fluorescence with excitation at $295 \mathrm{~nm}$ and emission at $395 \mathrm{~nm}$. The content of each cross-link was expressed as $\mathrm{mol} / \mathrm{mol}$ of collagen. The amount of collagen in the tissue was calculated by hydroxyproline assay using an elution procedure. The ratio of DHLNL/HLNL was assessed as a surrogate marker of the degree of hydroxylation of lysine in the collagen molecules. This ratio is highly regulated and distinctive for different collagenous tissues.

\section{Statistical analysis}

All values are listed as means with their standard deviations (mean $\pm S D$ ) in the text and the tables. Statistical significance was determined by one-way analysis of variance (ANOVA) with post hoc 
adjustment for multiple comparisons and was established at a $p$ value $<0.05$. All statistical analyses were performed using statistical analysis software (JMP V.3.1.6, SAS Institute, Cary, NC).

\section{Results}

\section{Histological findings}

\section{4 weeks after surgery $(n=4)$}

In the control limbs the interface between tendon and bone was filled fibrous tissue and inflammatory cells. In contrast, ingrowth of fibrous tissue and bone tissue in the TW (titanium-web) was found in experimental limbs. Villanueva's Goldner stain showed that calcified bone formation was observed in 3 out of 4 samples (Fig. 5).

\section{5 weeks after surgery $(n=4)$}

In the control limbs the interface between tendon and bone was more mature than those at 4 weeks, and several Sharpey-like fibers were observed in the interface layer. In contrast, in the TW limbs, ingrowth of tendon-like soft tissue and mineralized bone tissue into the TW connected the bone tunnel and transplanted tendon directly, without intervening tissues. No chondrocytes were observed (Fig. 6).

\section{Biochemical findings $(n=6)$}

The total number of collagen cross-links (the sum of DHLNL, HLNL, PYD) of the transplanted tendon in the TW limbs $(0.060 \pm 0.013 \mathrm{~mol} / \mathrm{mol}$ of collagen) and the tendon-like tissue within the TW itself $(0.041 \pm$ $0.007 \mathrm{~mol} / \mathrm{mol}$ of collagen) were, although low compared with that of the original patellar tendon (0.107 $\pm 0.027 \mathrm{~mol} / \mathrm{mol}$ of collagen), significantly greater than that of the transplanted tendon in the control limbs $(0.024 \pm 0.007 \mathrm{~mol} / \mathrm{mol}$ of collagen) (Fig. 7a). DPD was not detected in the tendons.

The maturation of collagen cross-links (the ratio of mature to immature cross-links) of the tendon in TW limbs $(0.19 \pm 0.02)$ and the tendon-like tissue within TW itself $(0.13 \pm 0.01)$ also were less than that of the native PT $(0.34 \pm 0.10)$, but were significantly higher $(p<0.05)$ than that of the tendon in control limbs $(0.09 \pm 0.02)$ (Fig. 7b).

The DHLNL/HLNL ratio, which is an indicator of tissue specific collagen maturation, was significantly higher in control limb tendons $(11.4 \pm 3.5)$ than in native PT $(2.6 \pm 0.4)$. On the other hand, there was no significant difference in the DHLNL/HLNL ratio between the tendon in TW limbs (4.5 \pm 1.2 ), the tendonlike tissue in the TW $(4.6 \pm 0.9)$ and the native PT (Fig. 7c).

\section{Discussion}


The present study demonstrated that the use of TW (titanium-web) in ACL reconstruction surgery promoted formation and maturation of collagen cross-links in the grafted tendon, and also directly binding the bone and tendon tissue through the TW.

Rodeo et al.[22, 23] reported that tendon healing occurred by bone ingrowth into the fibrovascular interface tissue that initially formed between bone and tendon in a dog model. There was progressive mineralization of the interface tissue, with subsequent bone ingrowth into the outer tendon and incorporation of the tendon graft into surrounding bone. As fibrovascular organization matured, the collagen fibers that attached the tendon to bone resembled Sharpey fibers. Biomechanical testing demonstrated a progressive increase in tendon pull-out strength that correlated with the amount of bone ingrowth, mineralization, and maturation of the healing tissue over 12 weeks. Similarly, on the control limbs of this study, the interface tissue between the bone and the tendon was formed 4 weeks after surgery, and the formation of Sharpey-like fibers was observed 15 weeks after surgery suggesting indirect insertion. Other hand, on the TW limbs of this experiment, ingrowth of tendon-like soft tissue was found to be similar to tendon tissue by collagen analysis. Furthermore, calcified bone tissue began to form in the TW 4 weeks after surgery, and connected the bone and the transplanted tendon directly within 15 weeks after surgery. Although this insertion is different from direct insertions which consists of a fibrocartilage layer, it is histologically closer to the normal insertion compared to indirect insertions.

Process of "ligamentization" was proposed by Amiel et al in 1986 [24]. Both tendons and ligaments are composed of connective tissue primarily containing types I and III collagen, proteoglycans, and cells. However, their precise composition and arrangement of matrix macromolecules differ to provide the specific mechanical properties required [25]. For example, compared with tendons, ligaments contain cells with rounded nuclei, and have more type III collagen and proteoglycans, less total collagen, different collagen cross-link composition, and different distribution of collagen fibril diameters [25]. Amiel et al demonstrated that a patellar tendon transplanted into the rabbit knee to replace an excised ACL continuously developed into a substance similar to a normal ACL [24]. They reported that spindle-shaped fibroblasts and coarse fibrillar crimp in PT autografts converted to rounded fibroblasts and fine fibrillar crimp similar to the normal ACL under histological examination. Also, collagen cross-links analysis revealed that the tissue changed from the low DHLNL PT pattern to the high DHLNL pattern observed in normal ACL [24].

Collagen cross-links of collagen fibers in tendon, ligament and bone can be divided into two types: lysine hydroxylase and lysyl oxidase-controlled cross-links (enzymatic cross-links) and glycation or oxidationinduced advanced glycation end products (AGEs) cross-links [26]. Among collagen cross-links, enzymedependent collagen cross-links play an important role in the strength of collagen fibers and acts as a scaffold for cell differentiation [10, 21, 26-29]. Enzyme-dependent collagen cross-links also can be divided into reducible immature cross-links anchoring two collagen molecules (lysinonorleucine crosslinks) and nonreducible mature cross-links anchoring three collagen molecules (pyridinoline cross-links). The total number of enzyme-dependent cross-links (immature cross-links + mature cross-links) is controlled by lysyl oxidase and will not be formed to exceed its required biomechanical strength $[10,26$, 
28, 29]. Three structures (dihydroxy-lysinonorleucine: DHLNL, hydroxy-lysinonorleucine: HLNL, and lysinonorleucine: LNL) have been identified by the difference of lysine hydroxylation rate in the immature cross-links. Identically, in the mature cross-links, two structures (pyridinoline: PYD, and deoxypyridinoline: DPD) have been identified. The component ratio of the five enzyme dependent cross-links (cross-links pattern) is considered to be tissue-specific. Skin, tendon, ligament, and bone types were identified by cross-link patterns using the ratio of high hydroxide to low hydroxide cross-links (a DHLNL/HLNL ratio or a PYD/DPD ratio) [10, 21, 26-29]. The change of collagen cross-links after ACL reconstruction in human has been considered. The autologous tendon implanted in the joint is initially subjected to a temporary lack of blood flow, reducing cellular synthesis of collagen. Thereafter, blood circulation is restored, promoting collagen synthesis, leading to collagen content increase, which results in increased immature cross-links and collagen fiber formation. Notably, the collagen fibers formed are arranged correctly. As mechanical load is applied, immature cross-links are converted to mature cross-links. Finally, collagen cross-links pattern converted from the tendon type (DHLNL/HLNL ratio $<1$ ) seen in semitendinosus, gracilis and patellar tendons into the ligament type (DHLNL/HLNL ratio >1) in the native ACL, is observed within one year postoperatively [10].

In this study we examined how the TW affects the biochemical ligamentization process from the early to middle stage. The total number of collagen cross-links of transplanted tendon in TW limbs and the tendon-like tissue within the TW itself were, although low compared with that of the original patellar tendon, significantly greater than that of the transplanted tendon in control limbs (Fig. 7a). Likewise, the maturation of collagen cross-links (the ratio of mature to immature cross-links) of the transplanted tendon and the tendon-like tissue within the TW were significantly greater than that of the transplanted tendon in the control limbs (Fig. 7b). These results suggested that the use of TW can promote the maturation and formation of collagen cross-links in the tendon 15 weeks after ACL reconstruction. For tissue specificity analysis, the DHLNL/HLNL ratio in the tendon parenchyma of control limbs increased significantly. In contrast, the ratio in the tendon parenchyma and tendon-like tissue in the TW maintained its tendon type pattern, similar to that of the original patellar tendon (Fig. 7c). This result suggested that TW does not adversely affect the collagen pattern.

There are some limitations to this study that must be addressed. First, the exact mechanism that promotes the formation of bone tissue and the maturation of the tendon tissue by the TW is still unclear, and warrants further consideration. Secondly, the formation of a fibrocartilaginous layer was not seen during the observed period. It is possible that a more natural structural regeneration of the tendon-bone junction may occur with more time. Adequate mechanical stresses and strong fixation are required to regenerate a firm tendon-bone junction, and it is possible that a stronger connection may be achieved by altering the shape and position of the TW. Thirdly, we did not perform biomechanical tests in the present study due to the limited number of samples. Further studies are needed to determine the breaking load and stiffness. Lastly, the procedure requires a relatively large bone tunnel compared to the graft tendon alone, because it is necessary to pass the transplanted tendon with the TW complex through the tunnel. When considering clinical application, damage to the meniscus or the transverse ligament can occur 
when porting a clinically sufficient thickness of the tendon, or bone tunnels can overlap in two-root reconstruction. These problems may be solved by improving the installation position of the TW.

\section{Conclusions}

We investigated the effectiveness of cylindrical TW (titanium-web) in tendon-bone interface healing and graft maturation in a miniature swine ACL reconstruction model. The present study suggested the following: (1) TW promoted ingrowth of tendon-like soft tissue and calcified bone tissue into TW connecting the bone and transplanted tendon directly within 15 weeks after surgery. (2) TW promoted the maturation and the formation of collagen cross-link in the tendon while maintaining the cross-link pattern of collagen at 15 weeks after surgery.

Our method using a clinically safe titanium material in a single surgery has potential clinical applications not only for ACL reconstruction, but also in cases in which the tendon insertion site has been destroyed by a tumor, or for tendon ruptures around the tendon-bone junction.

\section{Declarations}

\section{Ethics approval and consent to participate}

All experimental procedures were approved by the institutional animal care and use committee of the Jikei University (Tokyo, Japan) and were in compliance with the "Guide for the Care and Use of Laboratory Animals" and the ARRIVE (Animal Research: Reporting In Vivo Experiments) guidelines. The ID number of the approval was 2018-75.

\section{Consent for publication}

Not applicable.

\section{Availability of data and materials}

The datasets used and/or analyzed during the current study are available from the corresponding author on reasonable request. All data generated or analyzed during this study are included in this published article and its Additional file 1 .

\section{Additional file}

Additional file 1:

The raw data and the descriptive statistics data of this experiment.

\section{Competing interests}

The authors declare no competing interests. 


\section{Funding}

This work was supported by Japan Society for the Promotion of Science (JSPS) KAKENHI (Grant Number: JP25462352). The funder had no participation in the design of the study, data collection, data analysis, data interpretation and writing of the manuscript.

\section{Author' contributions}

$\mathrm{KR}, \mathrm{MS}$ and $\mathrm{KM}$ designed the experiments. KR, DK, SK, TO and HH performed the experiments. MS interpreted the data. KR, MS and TK wrote and edited the manuscript. All authors reviewed and approved the manuscript.

\section{Acknowledgments}

The authors gratefully acknowledge Ms. Kazumi Hirakawa and Ms. Rie Sawada (Reseach Assistants, Jikei University School of Medicine, Japan) for their technical assistance. Additionally, we thank the staff of the animal center of Hamri Co., Ltd for their care of the miniature swine and Dr. Azusa Seki for great support in surgical procedures.

\section{Abbreviations}

ACL: Anterior cruciate ligament

AGEs: advanced glycation end products

ANOVA: one-way analysis of variance

DHLNL: dihydroxy-lysinonorleucine

DPD: deoxypyridinoline

HLNL: hydroxy- lysinonorleucine

HPLC: high-performance liquid chromatography

LNL: Iysinonorleucine

MMA: methyl methacrylate

PT: patellar tendon

PYD: pyridinoline

rhBMP-2: recombinant human bone morphogenetic protein-2

TW: titanium-web 


\section{References}

1. Griffin LY, Albohm MJ, Arendt EA, Bahr R, Beynnon BD, DeMaio M, et al. Understanding and preventing noncontact anterior cruciate ligament injuries: A review of the Hunt Valley II Meeting, January 2005. Am J Sports Med. 2006;34:1512-32.

2. Lyman S, Koulouvaris P, Sherman S, Do H, Mandl LA, Marx RG. Epidemiology of anterior cruciate ligament reconstruction. Trends, readmissions, and subsequent knee surgery. J Bone Jt Surg - Ser A. 2009;91:2321-8.

3. Sánchez M, Anitua E, Azofra J, Prado R, Muruzabal F, Andia I. Ligamentization of Tendon Grafts Treated With an Endogenous Preparation Rich in Growth Factors: Gross Morphology and Histology. Arthrosc - J Arthrosc Relat Surg. 2010;26:470-80. doi:10.1016/j.arthro.2009.08.019.

4. Dong S, Xie G, Zhang Y, Shen P, Huangfu X, Zhao J. Ligamentization of Autogenous Hamstring Grafts after Anterior Cruciate Ligament Reconstruction: Midterm Versus Long-term Results. Am J Sports Med. 2015;43:1908-17.

5. Lu D, Yang C, Zhang Z, Xiao M. Enhanced tendon-bone healing with acidic fibroblast growth factor delivered in collagen in a rabbit anterior cruciate ligament reconstruction model. J Orthop Surg Res. 2018;13:1-8.

6. Takigami J, Hashimoto Y, Yamasaki S, Terai S, Nakamura H. Direct bone-to-bone integration between recombinant human bone morphogenetic protein-2-injected tendon graft and tunnel wall in an anterior cruciate ligament reconstruction model. Int Orthop. 2015;39:1441-7.

7. Atesok K, Fu FH, Wolf MR, Ochi M, Jazrawi LM, Doral MN, et al. Augmentation of tendon-to-bone healing. J Bone Jt Surg - Ser A. 2014;96:513-21.

8. Chen B, Li B, Qi YJ, Ni QB, Pan ZQ, Wang H, et al. Enhancement of tendon-to-bone healing after anterior cruciate ligament reconstruction using bone marrow-derived mesenchymal stem cells genetically modified with bFGF/BMP2. Sci Rep. 2016;6 February:1-9.

9. Everhart JS, Sojka JH, Kaeding CC, Bertone AL, Flanigan DC. The ACL injury response: A collagenbased analysis. Knee. 2017;24:601-7. doi:10.1016/j.knee.2017.01.013.

10. Marumo K, Saito M, Yamagishi T, Fujii K. The "ligamentization" process in human anterior cruciate ligament reconstruction with autogenous patellar and hamstring tendons: A biochemical study. Am J Sports Med. 2005;33:1166-73.

11. Lim JK, Hui J, Li L, Thambyah A, Goh J, Lee EH. Enhancement of tendon graft osteointegration using mesenchymal stem cells in a rabbit model of anterior cruciate ligament reconstruction. Arthrosc - $J$ Arthrosc Relat Surg. 2004;20:899-910.

12. Shahab-Osterloh S, Witte F, Hoffmann A, Winkel A, Laggies S, Neumann B, et al. Mesenchymal stem cell-dependent formation of heterotopic tendon-bone insertions (osteotendinous junctions). Stem Cells. 2010;28:1590-601.

13. Zhang X, Ma Y, Fu X, Liu Q, Shao Z, Dai L, et al. Runx2-modified adipose-derived stem cells promote tendon graft integration in anterior cruciate ligament reconstruction. Sci Rep. 2016;6 December 
2015:1-15. doi:10.1038/srep19073.

14. Kawakami Y, Takayama K, Matsumoto T, Tang Y, Wang B, Mifune Y, et al. Anterior Cruciate LigamentDerived Stem Cells Transduced with BMP2 Accelerate Graft-Bone Integration after ACL Reconstruction. Am J Sports Med. 2017;45:584-97.

15. Hashimoto Y, Yoshida G, Toyoda H, Takaoka K. Generation of tendon-to-bone interface "enthesis" with use of recombinant BMP-2 in a rabbit model. J Orthop Res. 2007;25:1415-24.

16. Yamazaki S, Yasuda K, Tomita F, Tohyama H, Minami A. The effect of transforming growth factor- $\beta 1$ on intraosseous healing of flexor tendon autograft replacement of anterior cruciate ligament in dogs. Arthrosc - J Arthrosc Relat Surg. 2005;21:1034-41.

17. Ross D, Maerz T, Kurdziel M, Hein J, Doshi S, Bedi A, et al. The Effect of Granulocyte-colony Stimulating Factor on Rotator Cuff Healing After Injury and Repair. Clin Orthop Relat Res. 2015;473:1655-64.

18. Kuboki Y, Furusawa T, Sato M, Sun Y, Unuma H, Abe S, et al. Bone enhancing effect of titaniumbinding proteins isolated from bovine bone and implanted into rat calvaria with titanium scaffold. Biomed Mater Eng. 2014;24:1539-48.

19. Li D, Iku S, Nemoto K, Kokai Y, Odajima T, Kuboki Y, et al. Geometry of artificial ECM ThreeDimensional Structure of Titanium-Web (TW) Promotes Differentiation of Human Bone Marrow Mesenchymal Cells into Osteoblasts. J Hard Tissue Biol. 2005;14:333-4.

20. Guo Z, Iku S, Mu L, Wang Y, Shima T, Seki Y, et al. Implantation with new three-dimensional porous titanium web for treatment of parietal bone defect in rabbit. Artif Organs. 2013;37:623-8.

21. Saito M, Marumo K, Fujii K, Ishioka N. Single-column high-performance liquid chromatographicfluorescence detection of immature, mature, and senescent cross-links of collagen. Anal Biochem. 1997;253:26-32.

22. Rodeo SA, Arnosczky SP, Torzilli PA, Hidaka C WR. Tendon-healing in a bone tunnel. 1993;75.

23. Rodeo SA, Suzuki K, Deng XH, Wozney J, Warren RF. Use of recombinant human bone morphogenetic protein-2 to enhance tendon healing in a bone tunnel. Am J Sports Med. 1999;27:476-88.

24. Amiel D, Kleiner JB, Roux RD, Harwood FL AW. The phenomenon of "ligamentization": anterior cruciate ligament reconstruction with autogenous patellar tendon. J Orthop Res. 1986;4:162-72.

25. Claes S, Verdonk P, Forsyth R, Bellemans J. The "ligamentization" process in anterior cruciate ligament reconstruction: What happens to the human graft? A systematic review of the literature. Am J Sports Med. 2011;39:2476-83.

26. Saito M, Marumo K, Soshi S, Kida Y, Ushiku C, Shinohara A. Raloxifene ameliorates detrimental enzymatic and nonenzymatic collagen cross-links and bone strength in rabbits with hyperhomocysteinemia. Osteoporos Int. 2010;21:655-66.

27. Fujii K, Yamagishi T, Nagafuchi T, Tsuji M, Kuboki Y. Biochemical properties of collagen from ligaments and periarticular tendons of the human knee. Knee Surgery, Sport Traumatol Arthrosc. 1994;2:229-33. 
28. Kato S, Saito M, Funasaki H, Marumo K. Distinctive collagen maturation process in fibroblasts derived from rabbit anterior cruciate ligament, medial collateral ligament, and patellar tendon in vitro. Knee Surgery, Sport Traumatol Arthrosc. 2015;23:1384-92.

29. Saito M, Marumo K. Collagen cross-links as a determinant of bone quality: A possible explanation for bone fragility in aging, osteoporosis, and diabetes mellitus. Osteoporos Int. 2010;21:195-214.

\section{Figures}

A

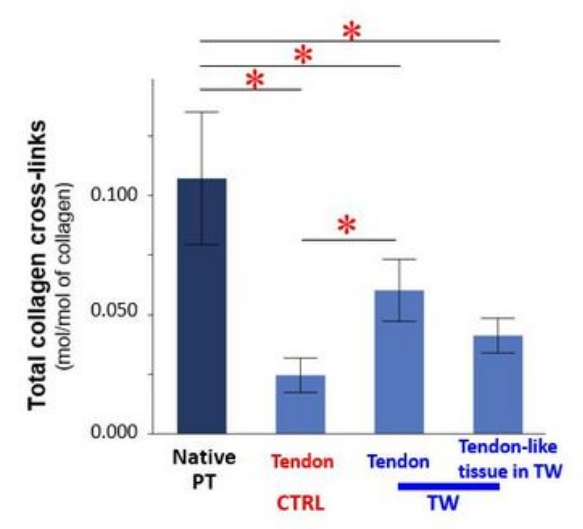

B

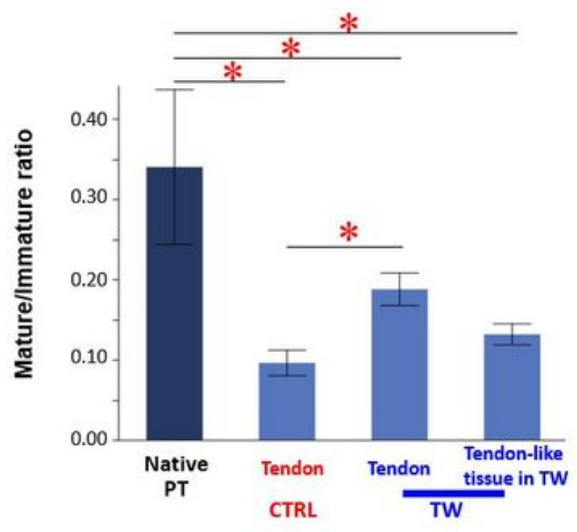

C

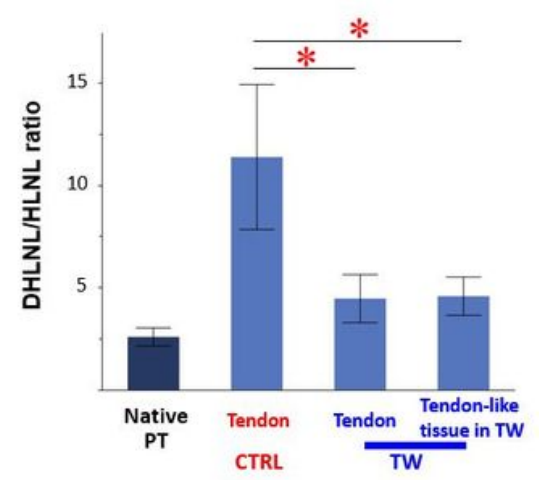

Figure 1

Implanted TW (titanium-web) in subcutaneous tissue. Collagen synthesis growing into the TW from surrounding soft tissue was observed. Bar scale $200 \mu \mathrm{m}$. 


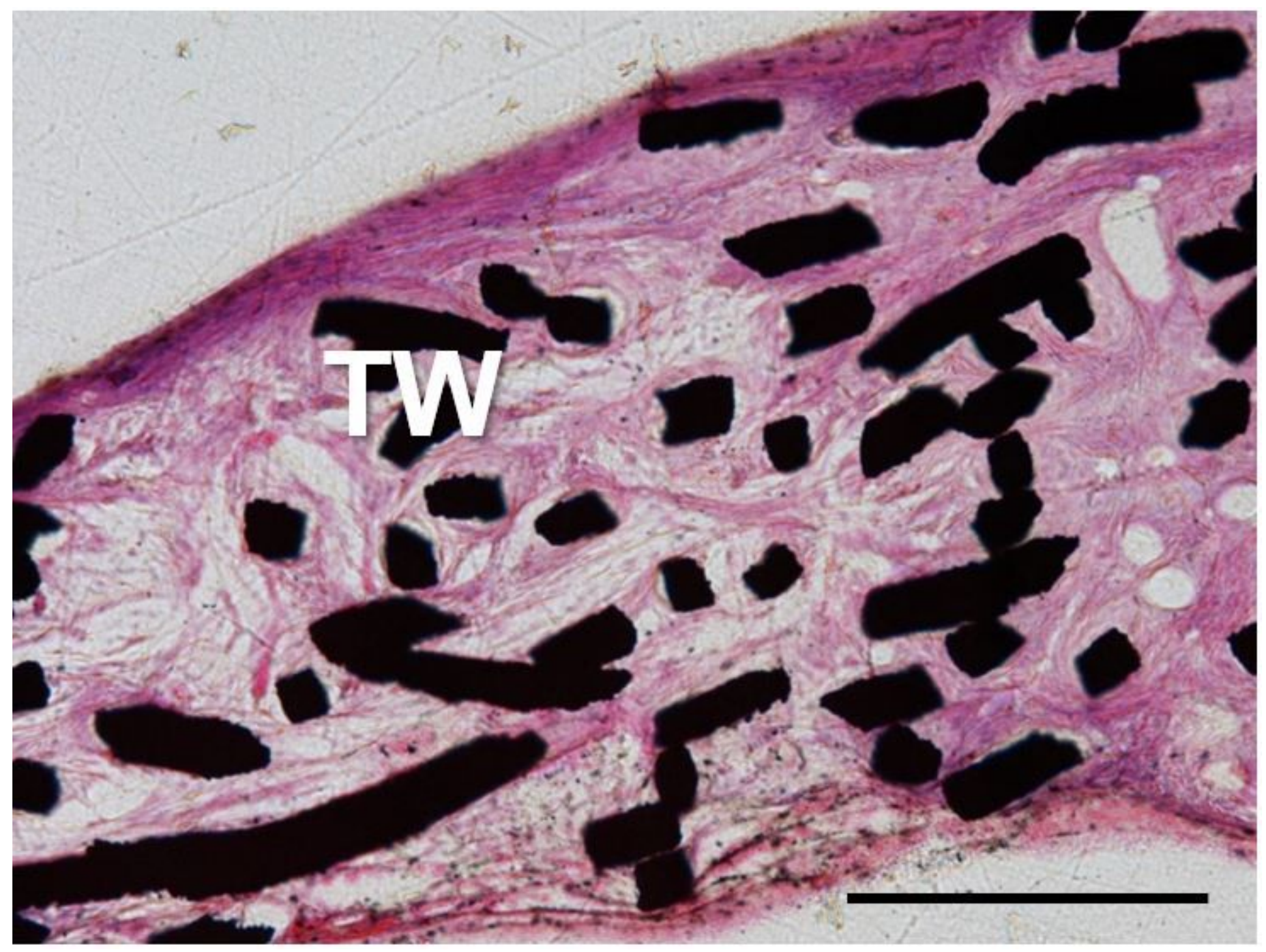

\section{Figure 2}

The cylindrical TW (titanium-web) consists of titanium fibers with a diameter of $50 \mu \mathrm{m} .(\mathrm{a}, \mathrm{b})$ The shape of the TW (outer diameter $6 \mathrm{~mm}$, inner diameter $3 \mathrm{~mm}$, length $10 \mathrm{~mm}$ ). (c) Titanium fibers with a diameter of $50 \mu \mathrm{m}$. 

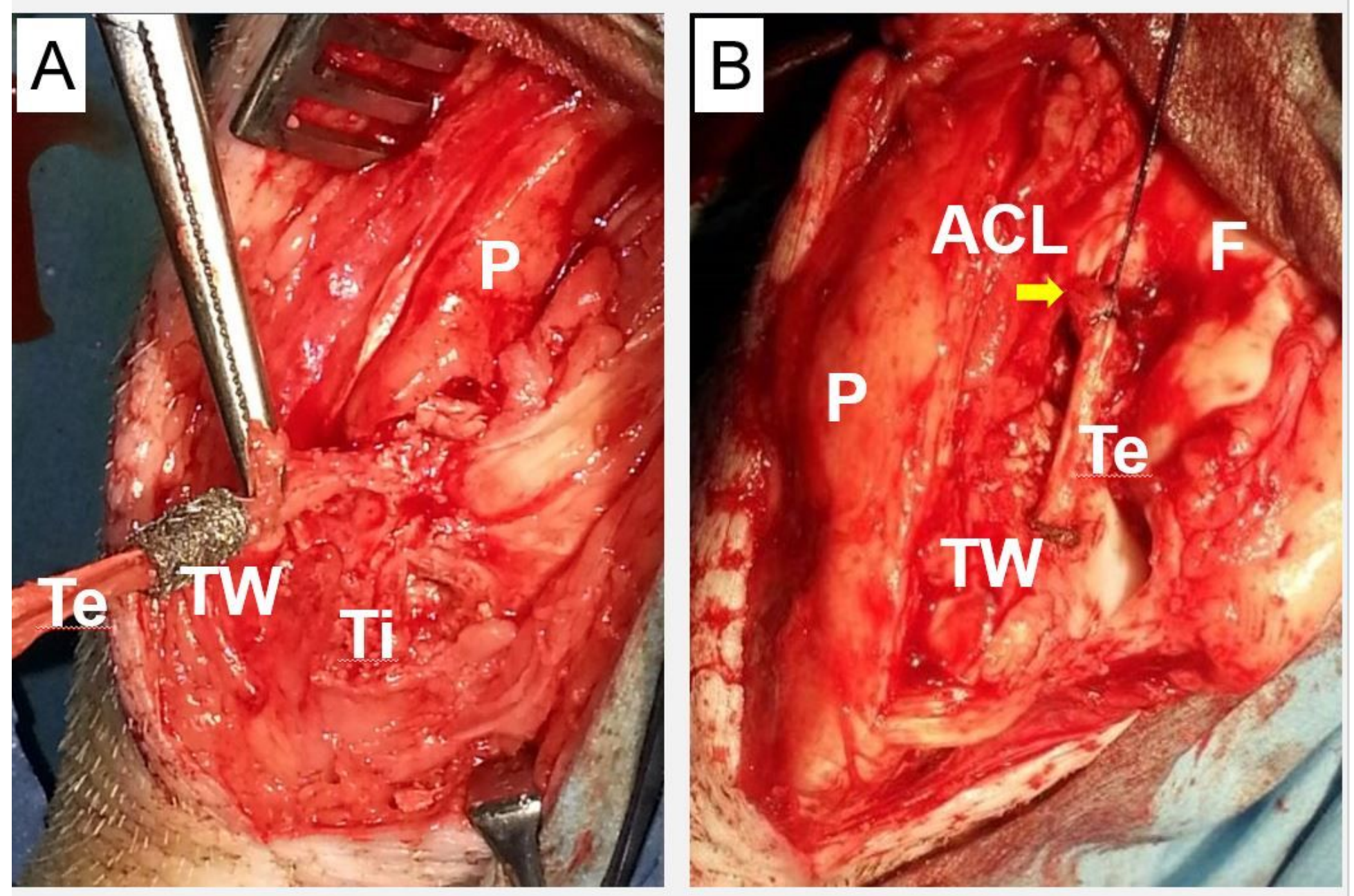

\section{Figure 3}

Bilateral ACL reconstruction with patellar tendon autograft. The tendon graft was inserted into the TW (titanium-web) (a) and the TW /tendon complex was placed into the tibial tunnel. The proximal side of the graft was sutured to the stump of the native ACL using a 4-0 nylon yarn (b). \{TW: titanium-web, F: femur, Ti: tibia, P: patella, ACL: the stump of the native ACL (arrow), Te: the grafted tendon\}. 
Control limb
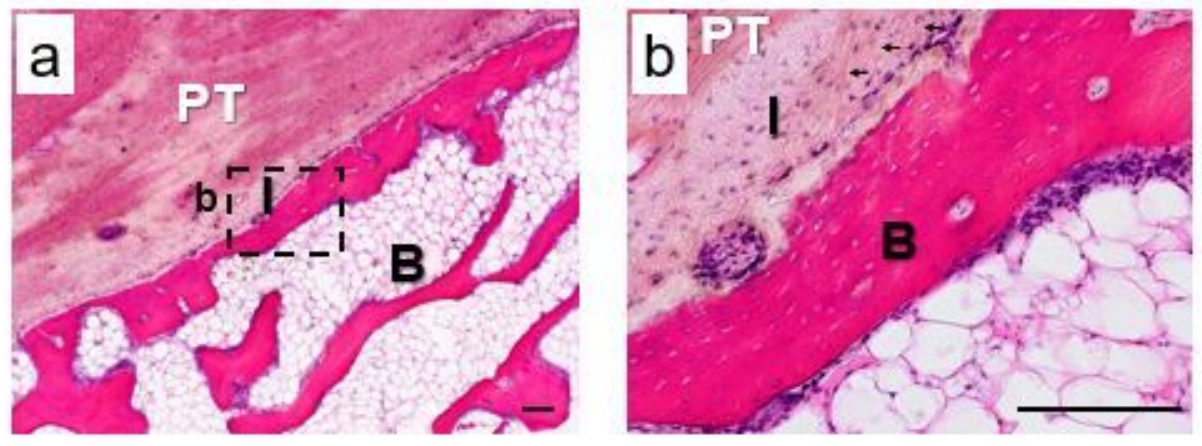

TW (Titanium-web) limb
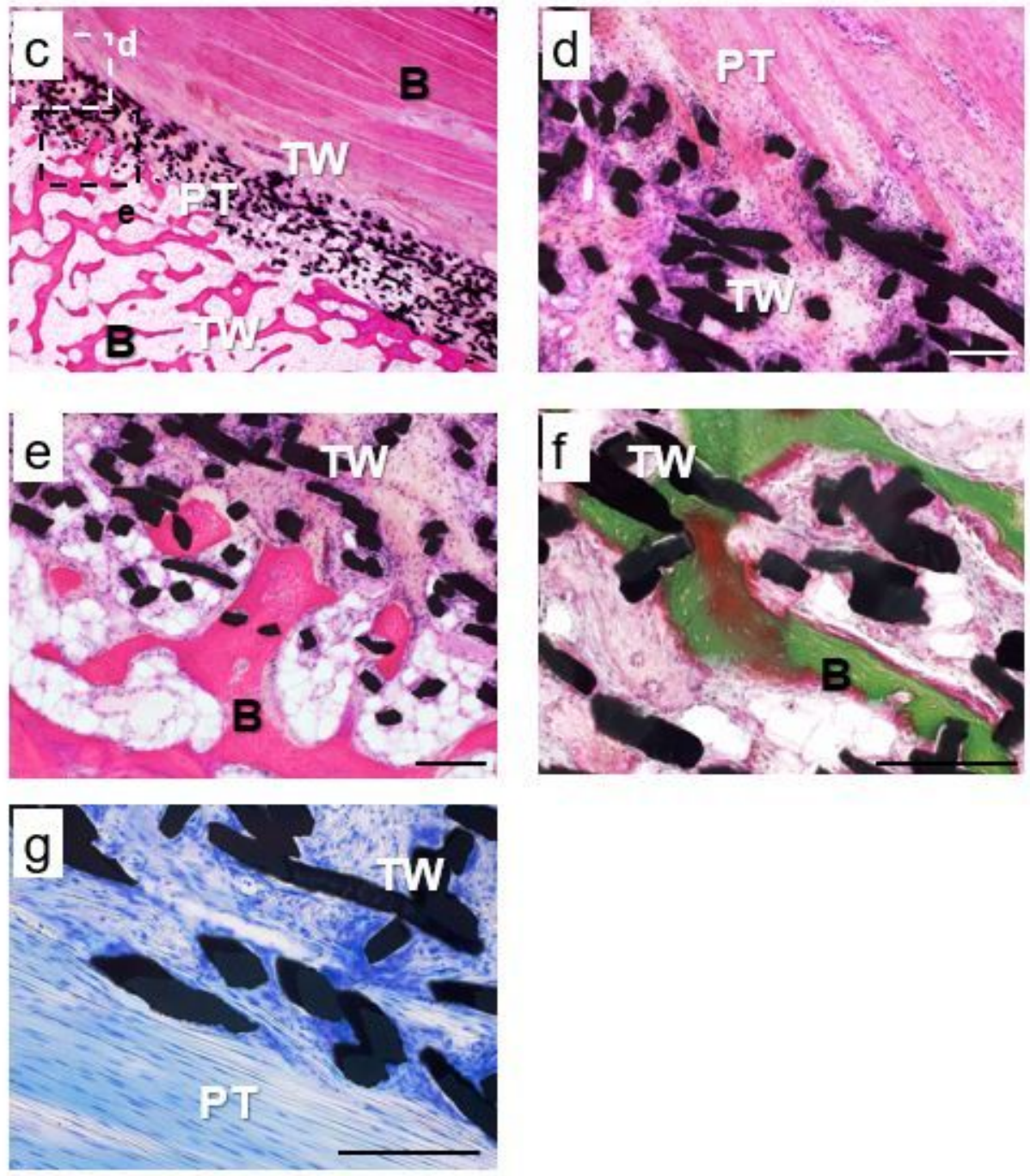

Figure 4

Bilateral anteroposterior radiographs of the knee after surgery. (a) TW (Titanium-web) limb. (b) Control limb. 


\section{Control limb}
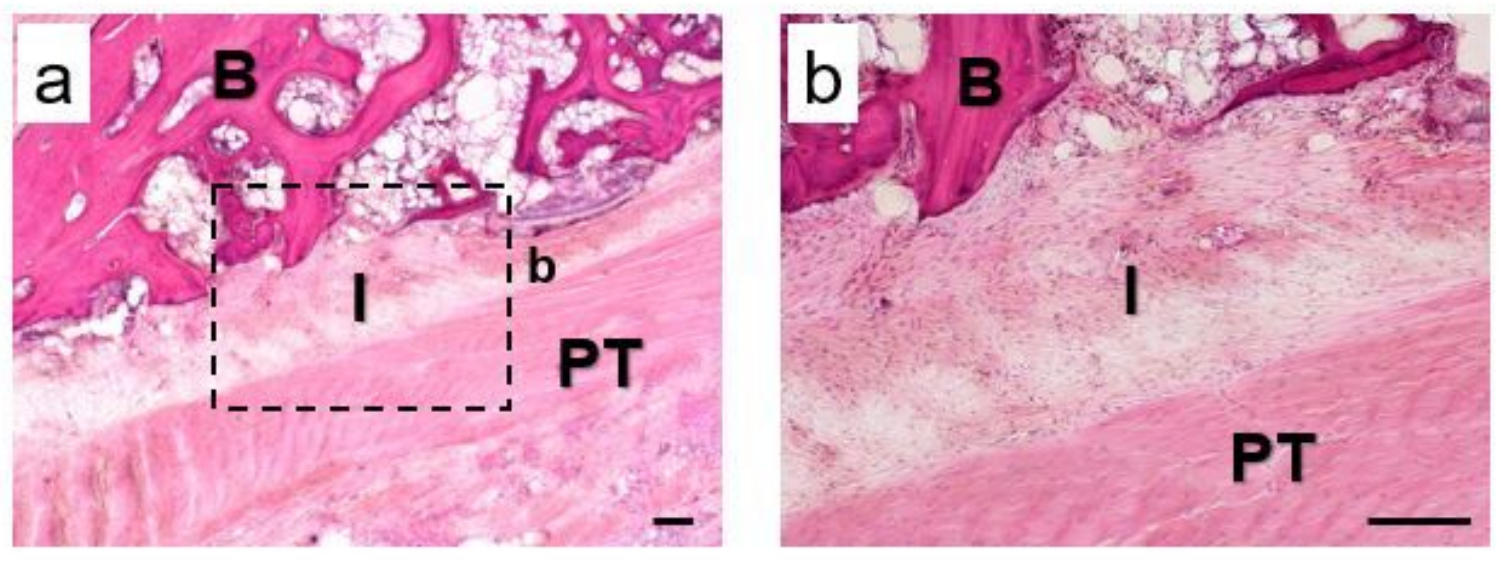

TW (Titanium-web) limb
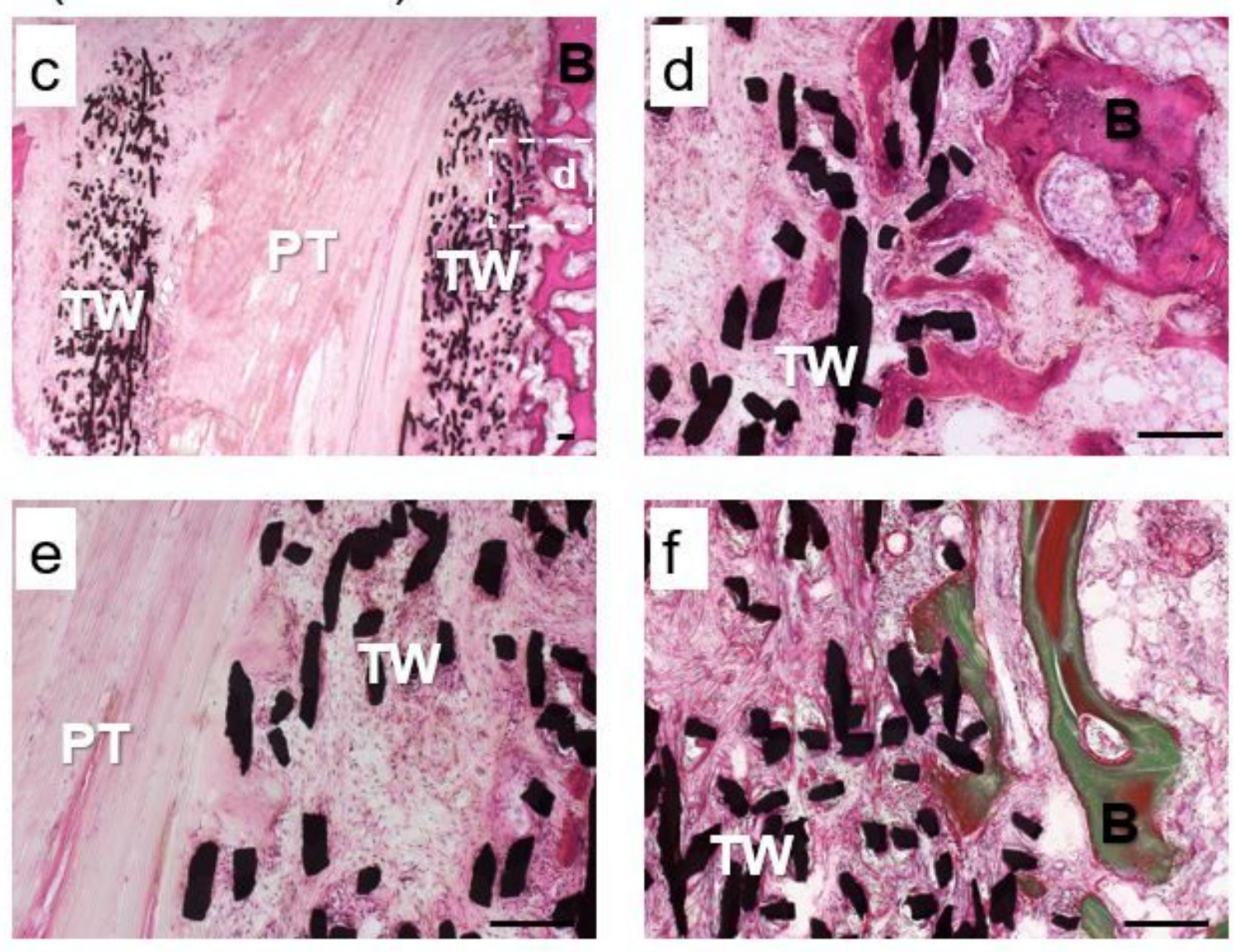

\section{Figure 5}

Histological examinations in control limbs $(a, b)$ and TW (titanium-web) limbs (c-e) 4 weeks after surgery. (a) The interface tissue was observed between the tendon and bone (H.E. staining, 4x). (b) Highmagnification (10x) view of the boxed area in the column of (a). Fibrous tissue and inflammatory cells were confirmed in the interface between the tendon and bone. (c) Low magnification view in TW limbs. (H.E. staining, 2x). (d) High-magnification (x) view of the boxed area in the column of (c). Ingrowth of 
fibrous tissue and bone tissue in the TW was observed. (H.E. staining, 10x). (e) Villanueva's Goldner staining. Calcified bone formation was confirmed in TW (10x). B: bone. I: interface tissue. PT: patellar tendon. TW: titanium-web. Bar scale $200 \mu \mathrm{m}$.
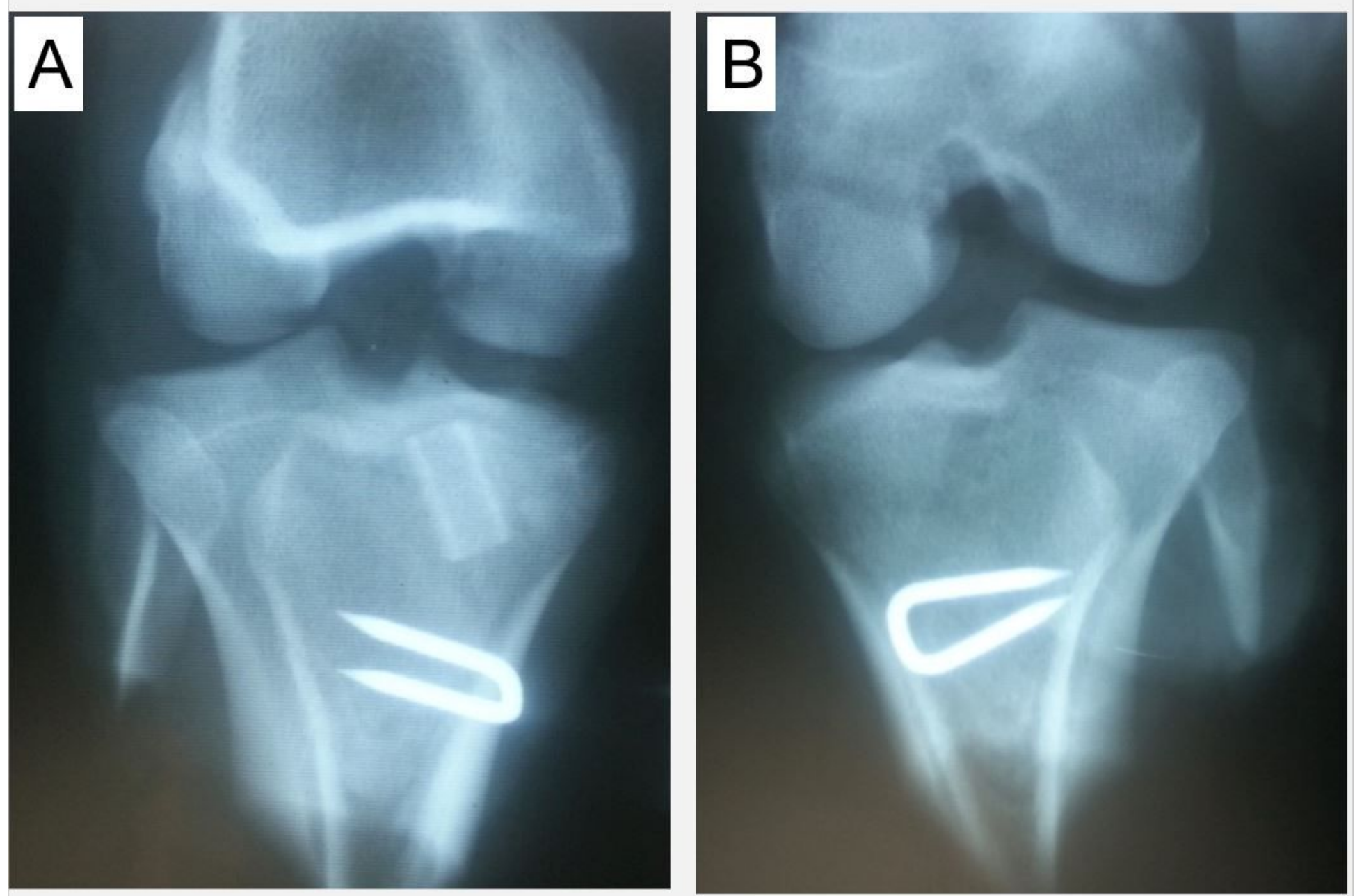

\section{Figure 6}

Histological examination in control limbs ( $a, b)$ and TW (titanium-web) limbs (c-g) 15 weeks after surgery. (a) The interface tissue between the tendon and the bone was more mature than at 4 weeks (H.E. staining, 4x). (b) High-magnification (20x) view of the boxed area in the column of (a). Several Sharpeylike fibers (arrows) were observed in the interface layer. (c) Low magnification view in TW limbs. (H.E. staining, 1.25x). (d) High-magnification (10x) view of the boxed area in the column of (c). Ingrowth of tendon and tendon-like soft tissue into the TW was more prominent than at 4 weeks. (e) Highmagnification (10x) view of the boxed area in the column of (c). Tendon-like soft tissue and bone tissue in TW connected directly. (f) Villanueva's Goldner staining. Calcified bone formation was confirmed in TW (20x). (g) Toluidine blue staining. Fibrocartilage tissue was not observed (20x). B: bone. I: interface tissue. PT: patellar tendon. TW: titanium-web. Bar scale $200 \mu \mathrm{m}$. 

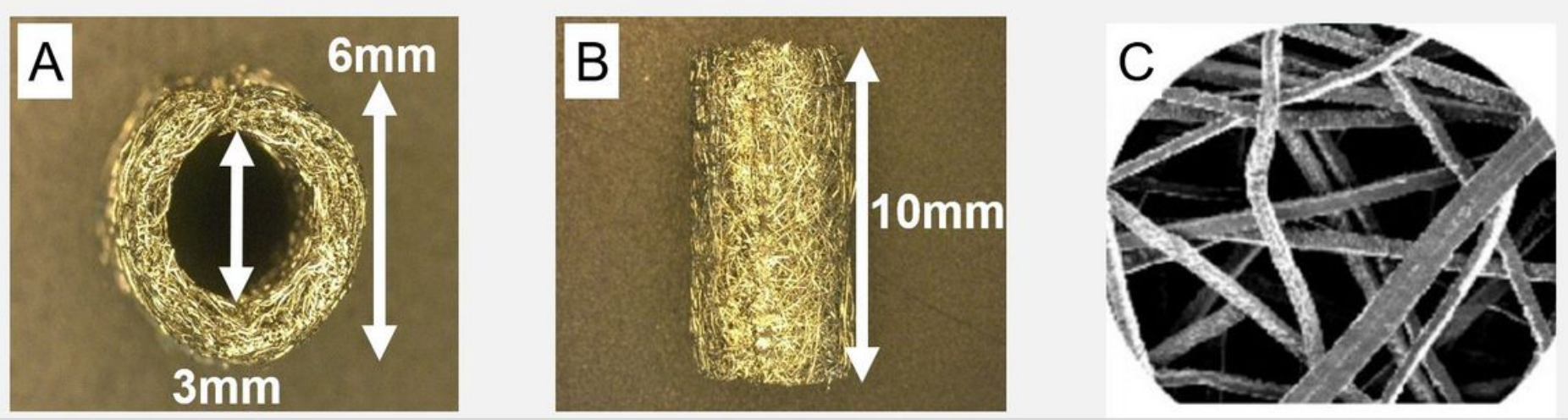

Figure 7

Quantitative analysis of collagen cross-links. (Native PT: native patellar tendon, CTRL: control limb, TW: titanium-web limb). (a) Total amount of collagen cross-links. (b) Maturation of collagen cross-links (the ratio of mature to immature cross-links). (c) The DHLNL/HLNL ratio in the matrices. ${ }^{*} p<0.05$

\section{Supplementary Files}

This is a list of supplementary files associated with this preprint. Click to download.

- RawdataofTW.docx

- NC3RsARRIVEGuidelinesChecklistryu.pdf 